\title{
A Metastatic Renal Carcinoid Tumor Presenting as Breast Mass: A Diagnostic Dilemma
}

\author{
Farnaz Hasteh, M.D., ${ }^{1}$ Robert Pu, M.D., Ph.D., ${ }^{2}$ and Claire W. Michael, M.D. ${ }^{2 *}$
}

We present clinicopathological and cytological findings of a well-defined breast mass in a patient with history of primary renal carcinoid tumor. Fine-needle aspiration (FNA) cytology showed monotonous tumor cells with plasmacytoid appearance arranged singly and in small clusters. Occasional tumor cells were arranged in acinar architecture resembling glandular differentiation. Tumor cells showed fine speckled chromatin. The unusual location for metastasis of this rare type of carcinoid tumor and overlapping cytological features with primary mammary carcinoma led to an erroneous preliminary cytological diagnosis of primary breast carcinoma with plasmacytoid features. Tumor cells in the corresponding cell block showed strong diffuse positivity for synapthophysin and pan-cytokeratin with weak focal positivity for chromogranin markers. These patterns of immunostaining were similar to the original renal carcinoid tumor. To the best of our knowledge, a few cases of carcinoid tumor metastatic to the breast have been reported in the literature and more than half of these cases were initially misdiagnosed as primary breast carcinoma causing unnecessary surgical treatment. This is a first reported case of metastatic renal carcinoid tumor into breast diagnosed with FNA biopsy. This report highlights the cytological features of well-differentiated neuroendocrine tumor (carcinoid tumor) and its potential diagnostic pitfalls. Diagn. Cytopathol. 2007;35:306-310. @ 2007 Wiley-Liss, Inc.

Key Words: carcinoma; neuroendocrine; carcinoid; breast; metastases

Neuroendocrine tumors are a family of neoplasms with wide range of morphologic, functional, and behavioral characteristics. These neoplasms originate from endocrine

\footnotetext{
${ }^{1}$ Department of Pathology, University of California, San Diego, California

${ }^{2}$ Department of Pathology, University of Michigan Medical Center, Ann Arbor, Michigan

*Correspondence to: Claire W. Michael, M.D., University of Michigan, 1500 E. Medical Center Dr., Room 2G332/Box 0054, Ann Arbor, MI 48109. E-mail: clairemi@med.umich.edu

Received 5 January 2006; Accepted 1 December 2006

DOI 10.1002/dc.20631

Published online in Wiley InterScience (www.interscience.wiley.com).
}

glands, nerve elements, or the diffuse endocrine system. They produce biologically active amines and peptide hormones. These tumors show evidence of neuroendocrine differentiation by variable positive staining for neruroendocrine markers such as synapthophysin and chromogranin. The epithelial type neuroendocrine tumors also show positive staining for pan-cytokeratin marker in a perinuclear dot-like pattern. Characteristically, these tumors show some similar cytological features regardless of the primary organ of origin. The goal of this article is to report an unusual metastatic location for a rare case of neuroendocrine tumor. We also briefly discuss the cytological features of well-differentiated neuroendocrine tumor (carcinoid tumor) with its differential diagnostic pitfalls.

\section{Case Reports \\ Clinical history}

A 61-year-old African-American woman from an outside hospital was referred to our institution for a second opinion regarding a $2-\mathrm{cm}$ palpable mass, located in the upper outer quadrant of her right breast. She had a history of left nephrectomy 5 years ago for a primary renal carcinoid tumor. A fine-needle aspiration (FNA) biopsy of the mass was requested.

\section{Materials and Methods}

FNA biopsies were performed using 23-gauge needles. On each pass, a drop of aspirated material was transferred into a glass slide and was smeared with another slide. One slide was air-dried and stained with rapid Diff-Quick stain for on-site assessment of sample adequacy and preliminary diagnosis. The other slide was alcohol-fixed and brought back to the laboratory for Papanicolaou stain. Needle rinses in Cytolyt solution were made into paraffin cell-blocks, and $4 \mu \mathrm{m}$ sections were stained with 


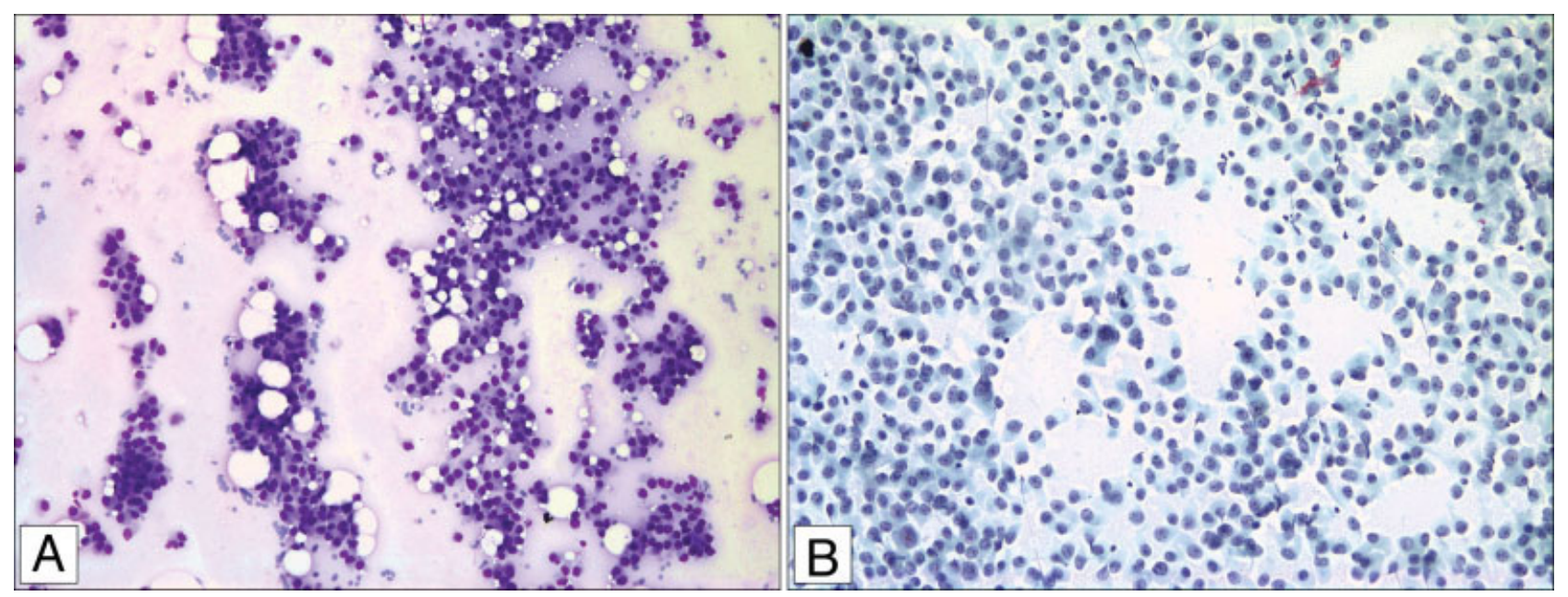

Fig. 1. A and B: FNA of breast mass show singly arranged and loose clusters of tumor cells with striking monotonous and plasmacytoid appearance (DQ and Papanicolaou stains, low power). [Color figure can be viewed in the online issue, which is available at www.interscience.wiley.com.]

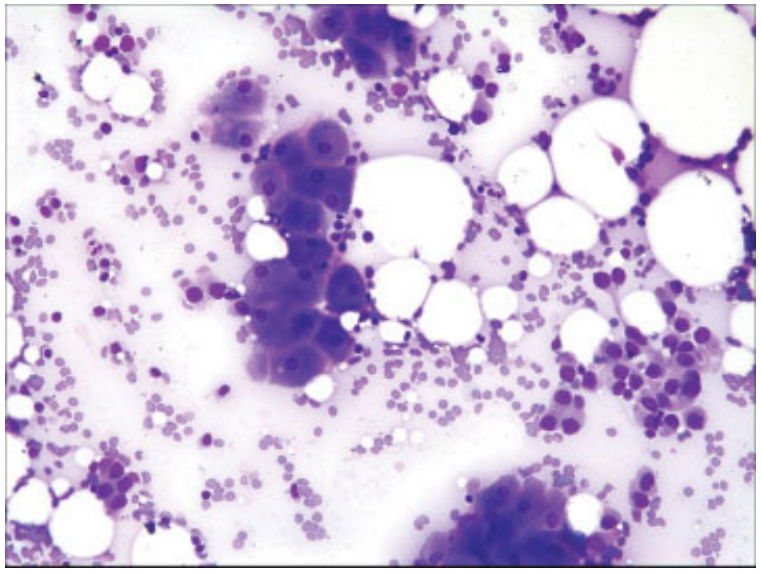

Fig. 2. Rare cluster of apocrine cells present with the tumor cells (DQ stain, high power). [Color figure can be viewed in the online issue, which is available at www.interscience.wiley.com.]

hematoxylin-eosin (H\&E). A total of five passes were performed with one pass taken only for the preparation of cell block and possible immunohistochemistry studies.

\section{Results}

\section{Cytological findings}

At low power microscopic magnification, the smears were cellular and composed of singly arranged and loose clusters of tumor cells with striking monotonous appearance (Fig. 1A). Benign appearing apocrine cells were seen mixed with tumor cells suggesting a fibrocystic background and the possibility of primary mammary origin of tumor (Fig. 2). The tumor cells had plasmacytoid appearance with eccentric nuclei and moderate amount of cytoplasm (Figs. 1A and B). The cytoplasm of tumor cells contained rare metachromatic granules in the Diff-Quick stain (Fig. 3). The nuclei of tumor cells had fine powdery

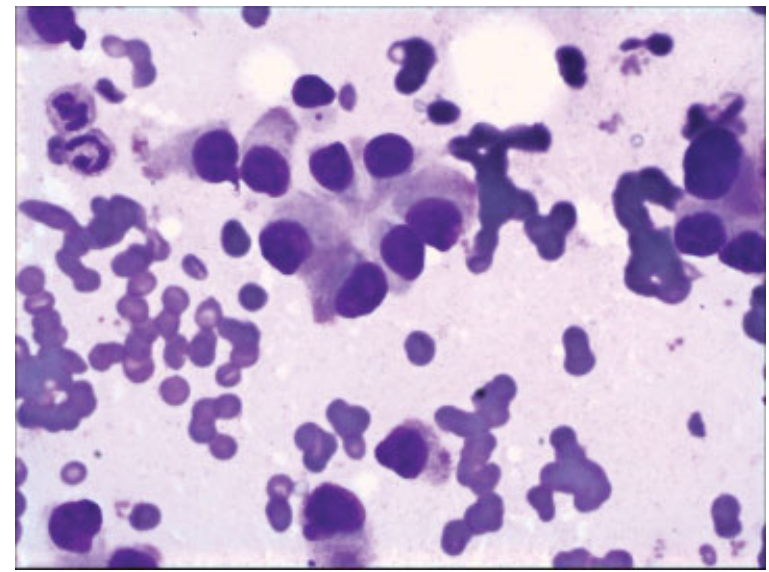

Fig. 3. Metachromatic granules present in cytoplasm of tumor cells (DQ stain, high power). [Color figure can be viewed in the online issue, which is available at www.interscience.wiley.com.]

chromatin with smooth nuclear membrane and small distinct nucleoli. Occasional tumor cells arranged in acinar architecture resembling glandular differentiation with few cells contained cytoplasmic vacuoles suggesting mucin production (Fig. 4). Small size blood vessels were not identified. The background was clean with no tumor cell necrosis or mitotic activity. On site preliminary diagnosis of primary mammary carcinoma with plasmacytoid features with differential diagnosis of metastatic renal carcinoid tumor was made pending further evaluation. Consequently, upon examining the alcohol-fixed, Papanicolaou-stained smears, the neoplastic cells showed more classical features of neuroendocrine differentiation with fine speckled or salt and pepper chromatin and occasional rosette formation (Fig. 4). These features supported the diagnosis of a neuroendocrine tumor, likely metastatic from primary renal carcinoid tumor. 


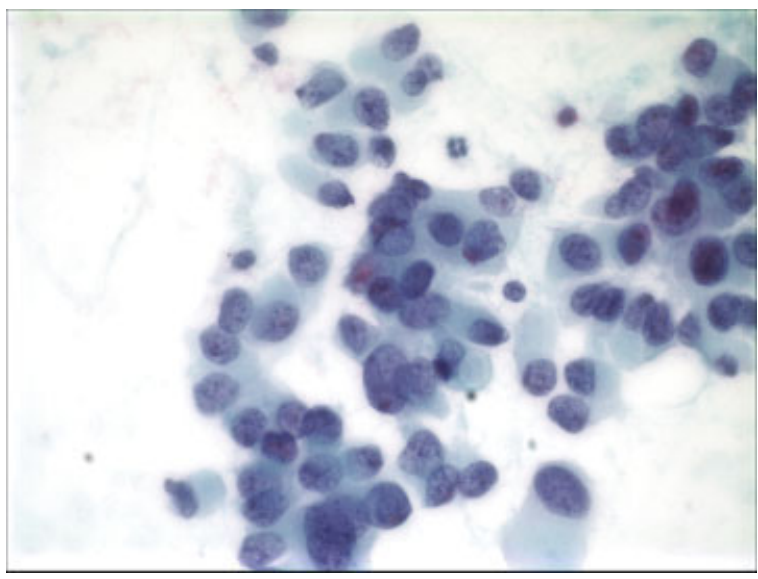

Fig. 4. Tumor cells show salt and peppery chromatin with rare cytoplasmic vacuole (Papanicolaou stain, high power). [Color figure can be viewed in the online issue, which is available at www.interscience. wiley.com.]

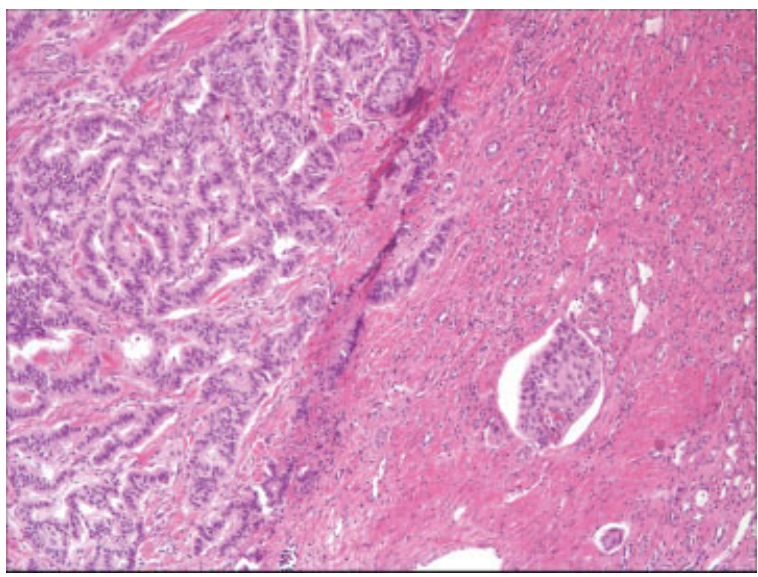

Fig. 5. Renal carcinoid tumor with angiolymphatic invasion $(H \& E$ stain). [Color figure can be viewed in the online issue, which is available at www.interscience.wiley.com.]
A representing section of the original renal carcinoid slide was also reviewed upon request from outside institute. Tumor showed classic features of typical carcinoid tumor in any other organ with extensive angiolymphatic and perirenal invasion (Fig. 5).

\section{Immunohistochemistry findings}

Immunohistochemistry (IHC) studies were performed on the corresponding cell block to explore the immunoreactivity of tumor cells for cytokeratin, hormonal receptors, and neuroendocrine markers. The pattern of staining were similar to that the original renal carcinoid tumor with diffuse strong synapthophysin positivity (Fig. 6A) and weak focal positivity for chromogranin. Tumor cells also showed strong diffuse positivity for another neuroendocrine marker (CD 56) (Fig. 6B). No immunoreactivity was seen for gross cystic disease fluid protein-15 (GCDFP-15), ER, and PR markers. In contrast, the benign apocrine cells showed positive staining for GCDFP-15. These immunophenotypic features were also supportive for the diagnosis of metastatic renal carcinoid tumor (see discussion).

No tissue biopsy was obtained and follow-up CT scan also showed widespread metastatic disease with multiple liver and bone metastasis. Currently, the patient is under special chemotherapy regimen (Sandtropin) for metastatic carcinoid tumor with scheduled follow-up imaging studies after the completion of treatment.

\section{Discussion}

The most common primary sites for metastatic carcinoid tumor are gastrointestinal (ileum), pulmonary tract, and pancreas. ${ }^{1}$ This tumor rarely occurs in kidney, with about 50 reported cases in the literature. ${ }^{2}$ A significant number of these patients with metastatic disease had a poor prognosis. $^{2}$

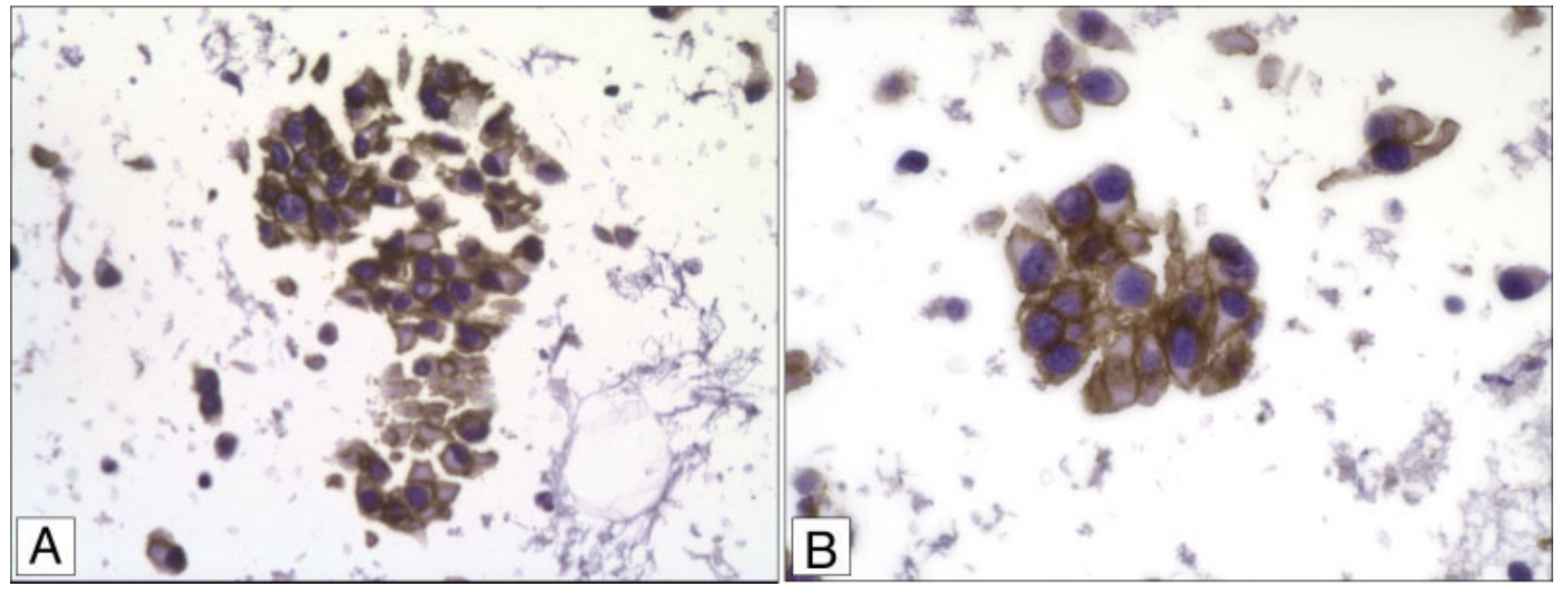

Fig. 6. A and B: Tumor cells show strong positivity for synaptophysin and CD56 (cell block section). [Color figure can be viewed in the online issue, which is available at www.interscience.wiley.com.] 


\section{METASTATIC RENAL CARCINOID TUMOR AS BREAST MASS}

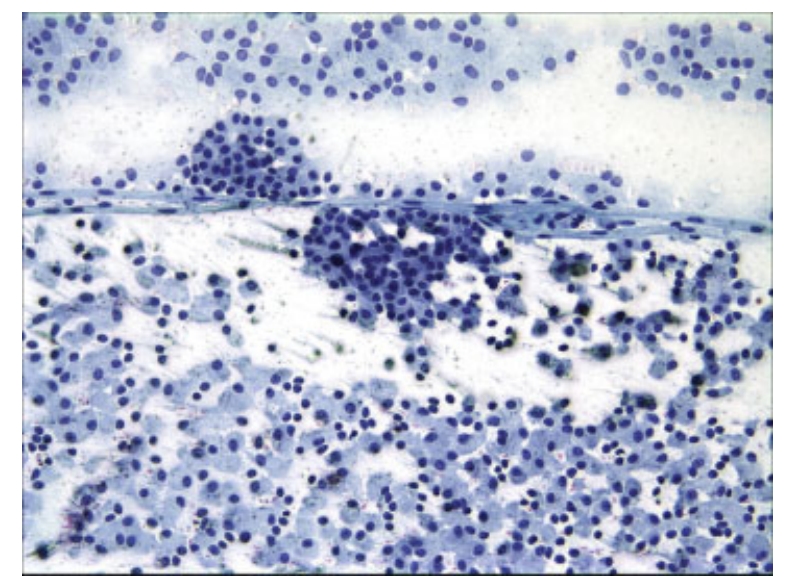

Fig. 7. Endobronchial carcinoid tumor with tumor cells around small sized blood vessels (Papanicolaou stain, high power). [Color figure can be viewed in the online issue, which is available at www.interscience. wiley.com.]

The common metastatic sites of general carcinoid tumor diagnosed by FNA biopsy include liver, lymph node, and the retroperitoneum. ${ }^{3}$ Rare cases of metastatic carcinoid tumor to the breast have been reported in the literature with majority of these cases primarily misdiagnosed as primary breast carcinoma causing unnecessary surgical treatment. $^{4-9}$ To the best of our knowledge, this is the first case of metastatic renal carcinoid tumor, which is diagnosed by fine needle aspiration biopsy.

While the cytological features of carcinoid tumor/well differentiated neuroendocrine tumor (WDNET) have been described in the literature, the potential diagnostic pitfalls of such diagnosis when it manifests as a metastasis to other organs such as breast are not adequately described.

FNA biopsies of carcinoid tumor/WDNET in general are cellular. Tumor cells are usually arranged singly, in loose clusters or trabecule. A helpful feature is the presence of feathery arrangement of tumor cells around small sized blood vessels (Fig. 7). The cells exhibit a plasmacytoid appearance with small to moderate amount of cytoplasm (Figs. 1A and B). Finding metachromatic granules in Diff-Quik stain is evidence of neuroendocrine differentiation (Fig. 3). The most reliable feature is presence of salt and pepper chromatin with or without small nucleoli that are best seen in Papanicolaou-stained smears (Fig. 4). Occasional acinar like or rosette formation of tumor cells are uncommon features in FNA biopsy, which can mimic glandular formation. Tumor cell necrosis and mitotic activity are usually absent in typical carcinoid tumor or WDNET.

The cytological features of our case as described previously showed clear evidence of diffuse neuroendocrine differentiation with cytoplasmic metachromatic granules, salt and pepper chromatin pattern, and monotonous appearance of tumor cells. However, unusual metastatic location for this renal carcinoid tumor, absence of feathery arrangement of tumor cells around blood vessels, occasional acinar arrangement of tumor cells, rare cytoplasmic vacuoles, and benign apocrine cells mixed with tumor cells raised the preliminary diagnosis of primary mammary carcinoma with plasmacytoid features and neuroendocrine differentiation.

Overall, the differential diagnosis for mammary carcinoma with plasmacytoid features includes primary adenocarcinoma with neuroendocrine differentiation, primary versus metastatic neuroendocrine (carcinoid) tumor, and invasive lobular carcinoma. Generally, lymphoproliferative disorders such as extranodal MALT lymphoma and malignant melanoma are also in the differential diagnosis because of cytological similarities especially the predominant pattern of single plasmacytoid cells.

Primary adenocarcinoma of breast with focal neuroendocrine differentiation should only show immunohistochemical expression of few cells with neuroendocrine markers. ${ }^{10}$ In addition, tumor cells show cytological features of adenocarcinoma with more nuclear overlapping and irregular nuclear borders. In contrast, primary neuroendocrine tumor of the breast is an uncommon carcinoma that usually occurs in older women with no history of primary carcinoid tumor in any other organs. ${ }^{10}$ According to the WHO classification of breast tumors, this specific terminology is used when more than $50 \%$ of tumor cells show positivity for neuroendocrine markers. ${ }^{10}$ Histologic grading and the stage of disease are the most important prognostic factors. The cytological and histological features of primary versus metastatic neuroendocrine carcinoma into the breast are similar. The presence of carcinoma in situ supports a primary mammary carcinoma. ${ }^{10}$ In addition, the primary breast neuroendocirne carcinoma usually shows immunoreactivity for neuroendocrine markers, GCDFP-15, and hormonal receptors. ${ }^{10,11}$ In contrast, the metastatic neuroendocrine carcinoma does not stain for the hormonal receptors and GCDFP-15.

Aspirate materials of primary classical lobular carcinoma of breast have low to intermediate cellularity with uniform population of small cells with moderate atypia and occasional cytoplasmic vacuoles or signet ring cells. The nuclei of lobular carcinoma do not show salt and pepper chromatin pattern. By immunostains, they show focal expression of neuroendocrine markers with positive staining for hormone receptors. ${ }^{12}$

FNA biopsy of malignant lymphoma shows monotonous population of single atypical cells with scant cytoplasm. The nuclei are more hyperchromatic and lymphoglandular bodies are usually seen in the background. Immunophenotypic features by immunostains or flowcytometry are helpful for diagnosis and classification of this tumor. 


\section{HASTEH ET AL.}

Finally the aspirates of metastatic malignant melanoma are quite cellular with many single cells with eccentric nuclei and distinct nucleoli and significant nuclear pleomorphism in contrast to monomorphic appearance of carcinoid tumor (WDNET). Occasional nuclear binucleation, intranuclear inclusion, and immunostaining patterns such as positive staining for HMB45 and S100 markers are supportive for diagnosis of malignant melanoma.

Overall, the FNA biopsy was a useful tool for the diagnosis of metastatic carcinoid tumor in our patient avoiding surgical excision and early initiation of chemotherapy regimen. In our case, the combination of cytomorphology and IHC results in conjunction with the patient past medical history enabled us to make a correct diagnosis and exclude the possibility of primary breast carcinoma.

\section{References}

1. Jackson SB, Williams HJ. Fine needle aspiration cytology of metastatic carcinoid tumor: Report of a case and review of the literature. Diagn Cytopathol 2003;28:49-53.

2. Begin LR. WHO organization classification of tumors. Tumors of urinary system. Geneva: World Health Organization; 2004. p 81-82.
3. Crapanzano JP. Cytology of low-grade endocrine neoplasms involving liver: A series of 24 specimens, including 4 with hepatoid or glandular features. Diagn Cytopathol 2004;30:31-38.

4. Fishman A, Kim HS, Girtanner RE, Kaplan AL. Solitary breast metastasis as first manifestation of ovarian carcinoid tumor. Gynecol Oncol 1994;54:222-226.

5. Rubio IT, Korourian S, Brown H, Cowan C, Klimberg VS. Carcinoid tumor metastatic to the breast. Arch Surg 1998;133:11171119.

6. Mosunjac MB, Kochhar R, Lau Sk, Mosunjac MI. Primary small bowel carcinoid tumor with bilateral breast metastases. Arch Pathol Lab Med 2004;128:292-297.

7. Kanthan R, Negreiros F, Kanthan SC. Colonic carcinoid metastatic to the breast. Arch Pathol Lab Med 2003;127:1373-1375.

8. Harrist TJ, Lalisher L. Breast metastasis: An unusual manifestation of the breast. Cancer 1977;40:3102-3106.

9. Lozowski JMS, Faregenburg D, Mishiriki Y, Lundy J. Carcinoid tumor metastatic to breast diagnosed by fine needle aspiration: Case report and literature review. Acta Cytol 1989;33:191-194.

10. Tavassoli FA, Devilee P. WHO classification of tumors. Tumors of female genital tract and breast. Geneva: WHO; 2004. p 22-25.

11. Saqi A, Oster MW, Vazquez MF. Mammary carcinomas with endocrine features. Diagn Cytopathol 2005;33:49-53.

12. Radhi JM. Immunohistochemical analysis of pleomorphic lobular carcinoma: Higher expression of p53 and chromogranin and lower expression of ER and PgR. Histopathology 2000;36:156-160. 